\title{
Sleep Quality Associated with the Onset of NAFLD: Suggested Hypotheses
}

\author{
Giovanni Tarantino
}

Department of Clinical

Medicine and Surgery,

Federico II University Medical School of Naples,

Naples, Italy

Address for correspondence: Giovanni Tarantino Department of Clinical Medicine and Surgery, Federico II University Medical School of Naples, Italy tarantin@unina.it

Received: 30.04.2019 Accepted: 01.05.2019
I read with great interest the paper by Okamura et al. [1] published in a recent issue of the Journal of Gastrointestinal and Liver Diseases, in which the authors for the first time showed that short sleep duration was a risk factor for incident non-alcoholic fatty liver disease (NAFLD). I found it very illuminating for its results and for the supposed mechanisms referred by the authors, but also for the possibility of more hypotheses that might explain their results. Nonalcoholic fatty liver disease is a very complex disease, about which much remains to be discovered regarding the origin, the evolution and mainly the therapy. Researchers are currently taking steps toward moving into human clinical trials based on findings in animal models. Nevertheless, it is noteworthy to stress that animal models of NAFLD do not completely mirror the human illness. With time, scientists expect that all obesity-related NAFLD patients will have treatment options.

To start with, obesity and overweight, playing a key role in the pathogenesis of NAFLD, were associated with short sleep duration. This relationship will be further analysed. But, what I need to stress is that any association, for its characteristics of bi-directionality, does not allow to draw strong conclusions and the dilemma, whether it is cause or effect, still remains.
In Okamura's study, both men and women with short sleep duration had higher BMI than the others.

Short sleep duration is generally associated with decreased leptin and increased ghrelin levels. Reduced leptin concentrations increase the appetite and lead to obesity. Augmented ghrelin production causes obesity by boosting dietary intake and lowering fat utilisation. Also, higher levels of inflammatory cytokines such as tumour necrosis factoralpha, and mainly interleukin- 6 were detected by fine research in subjects with a short sleep duration. This so-called chronic low-grade inflammation represents a central process in the pathogenesis of NAFLD.

Intriguingly, studying humans' short sleep duration was also reported to enhance the circadian misalignment on cortisol (perturbing the robustness of the circadian rhythm of cortisol) and also affecting the hypothalamic-pituitary-adrenal axis regulation, which could lead to subclinical hypercortisolism. Obviously, stress plays a determinant role in this dysfunctional process. The mechanism of hypercortisolism or increased loco-regional hypercotisolism is a compelling issue that tries to shed some light on the pathogenesis of NAFLD [2]. The dysregulation of the specific action of glucocorticoids (GC) and not of the alterations of GC levels has been proposed as a central feature of the metabolic syndrome, a universal presence in NAFLD patients [2]. Interestingly an overexpression of $11 \beta$-hydroxysteroid dehydrogenase type 1 in visceral adipose tissue and hepatic tissue was found [3]. However, further research is required to precisely define the role of GCs. It is necessary to stress that some diseases involving other organs are burdened by coexisting hepatic steatosis as a consequence of a prolonged steroid therapy.

An intriguing process linking short sleep duration and obesity-related NAFLD could be due to the gut flora dysbiosis. Again stress and hypothalamic-pituitary-adrenal axis are linked, clearly evidencing a gut-brain axis [4]. To tell the truth, now we are facing other organ-gut axis, such as gut-liver, hearth-gut, skin-gut, and gut-lung axis. The aforementioned links have an explanation based on the fact that gut microbiota can enhance local defence against enteral pathogens and is also believed to influence systemic immunity. Host-microbiome interactions represent inner catalysts of host physiology, while their dysregulation is implicated in a wide range of human diseases. The microbiome (bacteria, viruses and fungi) undergoes diurnal variation in composition 
and function, and this in turn drives oscillations in host gene expression and functions [5]. This aspect should be taken into consideration in various studies approaching gut flora microbiome. Coming back to precedent observations, population studies have identified an important dose-response relationship between short sleep duration, excess body weight and metabolic disturbances across all age groups and in several ethnic groups $[6,7]$.

Experimental studies in mice demonstrate that disturbed sleep is associated with gut microbiota composition. Experimental studies in humans find associations between curtailed sleep and measures of gut microbiota richness and diversity. In fact, authors assessed sleep metrics with indices of gut microbiome richness and diversity in a random general population sample of adults and found that better sleep quality and less sleepiness are significantly associated with greater species richness and diversity of the gut microbiota [8]. Could supplementation with probiotics revert this condition? A meta-analysis of randomised controlled trials [9] analysed the effects of probiotic supplementation on body weight, BMI, fat mass and fat percentage in subjects with overweight or obesity. It showed that administration of probiotics resulted in a larger reduction in body weight and fat percentage compared with placebo; however, the effect sizes were small. The effect of probiotics on fat mass was non-significant [9].

Recent data show that poor sleep quality and low sleep efficiency are associated with elevated levels of circulating inflammatory factors such as interleukin- 6 and $C$ reactive protein, but also with interferon gamma-inducible protein-10 in menopausal women. These findings provide further evidence that poor sleep quality is linked, from one side to low-grade systemic inflammation [10] and from another to the immune system imbalance, showing a critical role of this pivotal cytokine acting on interferon-gamma production. A fascinating information is that type 1 interferons are increased in obesity [11].

Still, convincing data suggest that endogenous interferonalpha may be a factor responsible for alterations of sleep, even though the impairing influence of interferon-alpha on sleep in humans is in contrast with findings of sleep promoting effects of this cytokine in animals [12]. To confirm the mediating effect of interferons-alpha, an up to date study [13] has added information about the cytokine network regulating the interplay between intramuscular triglycerides and obesity in obese patients with hepatic steatosis, emphasising the correlation between interferons and fat storage excess, and partially shedding light on the importance of these immune system constituents in obesity and obesity-related diseases.

\section{REFERENCES}

1. Okamura T, Hashimoto Y, Hamaguchi M, Obora A, Kojima T, Fukui M. Short sleep duration is a risk of incident nonalcoholic fatty liver disease: a population-based longitudinal study. J Gastrointestin Liver Dis 2019;28:73-81. doi:10.15403/jgld.2014.1121.281.alc

2. Tarantino G, Finelli C. Pathogenesis of hepatic steatosis: the link between hypercortisolism and non-alcoholic fatty liver disease. World J Gastroenterol 2013;19: 6735-6743. doi:10.3748/wjg.v19.i40.6735

3. Cooper MS, Stewart PM. 11Beta-hydroxysteroid dehydrogenase type 1 and its role in the hypothalamus-pituitary-adrenal axis, metabolic syndrome, and inflammation. J Clin Endocrinol Metab 2009;94:46454654. doi:10.1210/jc.2009-1412

4. Tetel MJ, de Vries GJ, Melcangi RC, Panzica G, O’Mahony SM. Steroids, stress and the gut microbiome-brain axis. J Neuroendocrinol 2018;30:e12548. doi:10.1111/jne.12548

5. Nobs SP, Tuganbaev T, Elinav E. Microbiome diurnal rhythmicity and its impact on host physiology and disease risk. EMBO Rep 2019;20:e47129. doi:10.15252/embr.201847129

6. Chaput JP, Brunet M, Tremblay A. Relationship between short sleeping hours and childhood overweight/obesity: results from the 'Quebec en Forme' Project. Int J Obes (Lond) 2006;30:1080-1085. doi:10.1038/sj.ijo.0803291

7. Hasler G, Buysse DJ, Klaghofer R, et al. The association between short sleep duration and obesity in young adults: a 13-year prospective study. Sleep 2004;27:661-666. doi:10.1093/sleep/27.4.661

8. Hagen WE, Holzhausen EA, Sethi KA, Malecki KM, Safdar N, Peppard PE. 0106 Sleep Duration and Quality and Diversity of the Gut Microbiome in a General Population Sample of Adults. Sleep 2019;42 Suppl 1:A43-A44. doi:10.1093/sleep/zsz067.105

9. Borgeraas H, Johnson LK, Skattebu J, Hertel JK, Hjelmesaeth J. Effects of probiotics on body weight, body mass index, fat mass and fat percentage in subjects with overweight or obesity: a systematic review and metaanalysis of randomized controlled trials. Obes Rev 2018;19:219-232. doi:10.1111/obr.12626

10. Huang WY, Huang CC, Chang CC, Kor CT, Chen TY, Wu HM Associations of Self-Reported Sleep Quality with Circulating Interferon Gamma-Inducible Protein 10, Interleukin 6, and High-Sensitivity C-Reactive Protein in Healthy Menopausal Women. PloS One 2017;12: e0169216. doi: 10.1371/journal.pone.0169216

11. Ghosh AR, Bhattacharya R, Bhattacharya S, et al. Adipose Recruitment and Activation of Plasmacytoid Dendritic Cells Fuel Metaflammation. Diabetes 2016;65:3440-3452. doi:10.2337/db16-0331

12. Späth-Schwalbe E, Lange T, Perras B, Fehm HL, Born J. Interferon-alpha acutely impairs sleep in healthy humans. Cytokine 2000;12:518-521. doi:10.1006/cyto.1999.0587

13. Tarantino G, Constantini S, Citro V, et al. Interferon-alpha 2 but not Interferon-gamma serum levels are associated with intramuscular fat in obese patients with nonalcoholic fatty liver disease. J Transl Med 2019;17:8. doi:10.1186/s12967-018-1754-6 